\title{
Formação académica em metodologia qualitativa: Prática pedagógica em Psicologia da Educação
}

\author{
Ana Pereira Antunes
}

\begin{abstract}
Resumo
O desenvolvimento das competências de investigação constitui uma preocupação na formação académica de futuros psicólogos. Associada a essa preocupação aparece ainda a discussão sobre a opção metodológica mais adequada à investigação: a metodologia quantitativa, a metodologia qualitativa ou mista. Com este estudo pretende-se, de uma forma mais específica, refletir e discutir sobre a adequação e eficácia do ensino de metodologia qualitativa, através da apresentação de uma Unidade Curricular de Métodos de Investigação. Participaram 12 alunos (10 mulheres e 2 homens), com uma média de idade de 25,4 anos, que frequentavam a disciplina Métodos de Investigação II, integrada no $1^{\circ}$ ano do plano curricular de um curso de $2^{\circ}$ ciclo em Psicologia da Educação, numa universidade portuguesa. Os dados foram recolhidos através de uma narrativa individual sobre o funcionamento da disciplina em estudo e foram analisados com recurso a análise de conteúdo, em função de 2 tópicos. Em relação ao tópico de análise sobre a Dinâmica das Aulas registaram-se categorias como: Clima de aula e motivação, Estratégias pedagógicas e Papel do Professor; e, em relação ao tópico Abordagem Qualitativa registaram-se categorias como: Esclarecimento e conhecimento, Rigor e validade, Experienciação e Ética. Os resultados traduzem, na generalidade, satisfação com a dinâmica das aulas bem como a aquisição de conhecimento e de competências de investigação qualitativa, revelando adequação e eficácia no ensino de investigação qualitativa. Tecem-se algumas considerações e apresentam-se linhas orientadores para trabalhos futuros.
\end{abstract}

Palavras-chave:

investigação qualitativa; prática pedagógica; competências de investigação; Psicologia da Educação 


\title{
Academic training in qualitative research: Teaching practice in Educational Psychology
}

\begin{abstract}
The development of research skills is a concern in the academic training of future psychologists. Associated with this concern, there is also a discussion about the most appropriate methodological option for research: quantitative, qualitative or mixed methodology. The main goal of this study is to reflect and to discuss, in a more specific way, the adequacy and the effectiveness of teaching qualitative research, through the presentation of a course of Research Methods. Participants were 12 students (10 women and 2 men), with a mean age of 25.4 years old, who participated in the course of Research Methods II, integrated in the $1^{\text {st }}$ year of the curricular plan of a $2^{\text {nd }}$ cycle course in Educational Psychology, in a Portuguese university. The data were collected through an individual narrative about the functioning of the course and were analyzed using content analysis, based on 2 topics. In the topic Dynamics of Classes were registered categories such as: Classroom climate and motivation, Pedagogical strategies and Teacher's role. In the topic Qualitative Approach there were categories such as: Clarification and knowledge, Rigor and validity, Experience, and Ethics. The results generally reflect satisfaction with the dynamics of the classes as well as the acquisition of knowledge and qualitative research skills, revealing adequacy and effectiveness of teaching qualitative research. Some considerations are discussed and guidelines for future work are presented.
\end{abstract}

Keywords: qualitative research; teaching practice; research skills; Educational Psychology

\section{Formation universitaire en méthodologie qualitative: Pratique pédagogique en Psychologie de l'Education}

Résumé: Le développement des compétences d'investigation représente une inquiétude au sein de la formation universitaire des futurs psychologues. Associée à cette préoccupation, surgit aussi la discussion à propos de l'option méthodologique la plus adaptée à l'investigation: la méthodologie quantitative, la méthodologie qualitative ou mixte. Cette étude vise, de façon plus spécifique, penser et discuter l'adéquation et l'éfficacité de l'enseignement de la méthodologie qualitative, à travers la présentation d'un cours de Méthodes d'Investigation. On a participé 12 étudiants (10 femmes et 2 hommes), avec une moyenne d'âge de 25,4 ans, qui fréquentaient le cours de Méthodes d'Investigation II, integré en 1ère année du deuxième cycle du cursus de Psychologie de L'Éducation, d'une Université Portugaise. Les données ont été recueillies à travers une narrative individuelle sur le fonctionnement de la matière en question et ont été analysées ayant recours à l'analyse du contenu, en fonction de 2 sujets. Au sujet de l'analyse de la Dynamique des Cours, ont été enregistrées les catégories suivantes: Ambiance de la classe et motivation, Stratégies pédagogiques et Rôle du professeur; quant au sujet de l'Abordage Qualitatif, ont été notées les catégories telles que: Clarification et connaissance, Rigueur et validité, Expérience, et Éthique. Les résultats attestent, en général, une satisfaction avec la dynamique des cours, ainsi que l'acquisition de connaissances et de compétences d'investigation qualitative, demontrant ainsi l'adéquation et l'éfficacité de l'enseignement de la méthodologie qualitative. Sont tissées quelques réflexions et présentées les lignes directrices pour de prochaines études.

Mots-clés: investigation qualitative; pratique pédagogique; compétences d'investigation: Psychologie de L'Education.

\section{Formación académica en metodología cualitativa: La práctica docente en Psicología de la Educación}

Resumen: El desarrollo de habilidades de investigación es una preocupación en la formación académica de los futuros psicólogos. En relación con esta preocupación persiste el debate sobre el enfoque metodológico más adecuado para la investigación: una metodología cuantitativa, la metodología cualitativa o mixta. Este estudio tiene por objeto, concretamente, reflexionar y debatir sobre la adecuación y la eficacia de la enseñanza de la metodología cualitativa a través de la presentación de un Curso de Métodos de Investigación. Participaron 12 estudiantes (10 mujeres y 2 hombres) con una edad media de 25,4 años, que asistieron al curso de Métodos de Investigación II, que forma parte del 1er año del plan de estudios de un curso de $2^{\circ}$ ciclo en Psicología de la Educación, en una universidad portuguesa. Los datos fueron recogidos a través de una narrativa personal sobre el funcionamiento del curso y se analizaron mediante el análisis de contenido, de acuerdo con dos temas. En cuanto al tema de la Dinámica de las Clases se registraron categorías tales como: Clima de classe y motivación, Estrategias pedadógicas y Papel del profesor; y, en relación con el tema Enfoque Cualitativo había categorías tales como: Clarificación y conocimiento, Rigor y validez, Experiencia, y Ética. Los resultados muestran, en general, satisfacción con la dinámica de las clases y la adquisición de conocimientos y habilidades de investigación cualitativa, revelando adecuación y eficacia de la enseñanza de la metodología cualitativa. Se discuten algunas consideraciones y se presentan directrices para trabajos futuros.

Palabras clave: investigación cualitativa; práctica docente; competencias de investigación; Psicología de la Educación 


\section{Introdução}

A investigação qualitativa, no campo da Psicologia, tem ocupado um lugar discreto, e até de alguma forma subalterno, em relação à investigação quantitativa (Povee \& Roberts, 2014; Roberts \& Povee, 2014). Todavia, tem-se registado um caminho de abertura, aceitação e integração da investigação qualitativa em várias áreas da Psicologia, reconhecendo-se a sua importância e contributo ao saber psicológico (Culver, Gilbert, \& Sparkes, 2012; Poulin, 2007; Rodríguez, Anguera, Suárez, León, \& Márquez, 2014).

Ao pensar-se na formação de futuros psicólogos, incluindo a formação académica não só o desenvolvimento de competências profissionais, mas também de investigação, coloca-se a questão do ensino da metodologia de investigação e, de uma forma mais específica e no âmbito deste trabalho, da investigação qualitativa. É bem verdade que, nos últimos anos, se têm registado oportunidades de formação nesse sentido, também em Portugal. Mas, também é verdade, que o paradigma dominante, na maioria das universidades, é o quantitativo (Povee \& Roberts, 2014; Roberts \& Povee, 2014) e as ofertas existentes, no ensino superior, se pautam por alguma inconsistência (Poulin, 2007). Por um lado, têm de ser consideradas questões institucionais, relacionadas com o paradigma privilegiado nas equipas de investigação, ou seja, se a prática aceite e defendida na universidade se pauta exclusivamente pelo positivismo ou se reconhece a pertinência da opção metodológica de investigação qualitativa, permitindo oferecer aos alunos essa possibilidade de formação (Cox, 2012). E, por outro lado, surge a questão de como poderá ser realizada essa formação já que, apesar de poder constituir uma experiência muito gratificante e agradável, coloca ao professor diversos desafios (Amado, 2014; Humble \& Sharp, 2012), pois terá sempre de enfrentar o dilema de como gerir e distribuir pelas atividades letivas o ensino dos conceitos e conteúdos de ordem ontológica e epistemológica, bem como as oportunidades para o desenvolvimento de competências práticas de recolha e análise de dados (Hansman, 2015).

De uma forma geral, o contacto dos alunos, dos cursos de Psicologia, com a metodologia qualitativa acontece depois de terem obtido conhecimento sobre a metodologia quantitativa, pelo que tendem a desenvolver uma série de crenças erradas sobre a abordagem qualitativa, crenças essas que alguns professores e investigadores positivistas partilham (Povee \& Roberts, 2014). Nesse sentido, nas aulas de metodologia qualitativa:

When we introduce students to the assumptions and values of qualitative research, we can start off back-footed; we also have to teach them that there is more than one way to do research within psychology, and that 
qualities such as subjectivity do not produce bias that undermines the research, but are essential to good qualitative research practice. (Clark \& Braun, 2013, pp. 120-121)

A experiência de investigação do docente nesta área revela-se importante, mas não suficiente, requerendo-se também a atualização permanente do conhecimento e a discussão com outros investigadores e com os próprios alunos (Amado, 2014). Contudo, é frequente encontrar-se docentes que lecionam esta disciplina com pouca formação sobre como conduzir este processo de ensino, sendo que, apesar da diversidade e quantidade de literatura acerca da metodologia de investigação qualitativa (e.g., Denzin \& Lincoln, 2005), a informação sobre como ensinar investigação qualitativa, nos seus mais variados tópicos, é bastante escassa (Hansman, 2015).

Nesse sentido, têm sido desenvolvidos alguns estudos mais recentemente, não só na área da Psicologia, procurando contribuir para a resposta a essa necessidade de orientação no modelo de ensino do docente/investigador qualitativo, ilustrando, a partir das próprias vivências e práticas, percepções e metodologias desenvolvidas (e.g., Clarke \& Braun, 2013; Cordner, Klein, \& Baiocchi, 2012; Cox, 2012; Goussinsky, Reshef, Yanay-Ventura, \& Yassour-Borochowitz, 2011; Hansman, 2015; Humble \& Sharp, 2012; Poulin, 2007; Reynolds-Keefer, 2010, Terkildsen \& Petersen, 2015). Ainda que não haja uma forma única de ensinar metodologia qualitativa (Goussinsky et al, 2011; Page, 1997), destaca-se a importância atribuída à epistemologia e às metodologias que permitem adquirir competências técnicas e práticas (Clark \& Braun, 2013; Hansman, 2015; Terkildsen \& Petersen, 2015). Neste processo, assumindo o investigador o papel de "instrumento" na recolha de dados importa considerar que

qualitative researchers must be reflective and cognizant of "who" they themselves as researchers are, and what their "stories" (autobiographies) are, and the meaning they make of the world, understanding that these are the lenses through wich they, as researchers, will view their research participants and interpret the phenomena being studied. (Hansman, 2015, p.277)

Nesse sentido, também os alunos deverão fazer esta aprendizagem e este reconhecimento pessoal, verificando-se, ao mesmo tempo, a adoção de uma prática pedagógica nos módulos de investigação qualitativa valorizando o "learning by doing" (Cordner et al, 2012).

Face ao exposto, este estudo decorre não só da revisão da literatura efetuada mas também da necessidade sentida, ao longo da prática pedagógica, de analisar a adequação e eficácia da lecionação de uma disciplina de metodologia de investigação qualitativa no âmbito da formação académica de futuros psicólogos. Assim, na 
sequência de um trabalho exploratório anterior (Antunes, 2016), pretende-se refletir sobre o ensino da metodologia qualitativa, apresentando uma Unidade Curricular (UC) específica, denominada, neste caso Métodos de Investigação II (MI II) e a apreciação do seu funcionamento, junto de um grupo de alunos de $2^{\circ}$ ciclo do processo de Bolonha.

\section{Método}

\section{Participantes}

O grupo de participantes foi constituído por 12 alunos (10 mulheres e 2 homens) com uma média de idade de 25,4 anos (oscilando entre 21 anos e 47 anos), os quais frequentavam $\circ 1^{\circ}$ ano de um curso de $2^{\circ}$ ciclo do processo de Bolonha, em Psicologia da Educação, numa Universidade Pública Portuguesa. Estes alunos pertenciam a uma turma de 17 alunos que, no ano letivo de 2015/2016, frequentaram a UC de MI II, integrante do plano curricular do referido curso.

\section{Materiais}

Para a realização deste trabalho utilizaram-se a narrativa e a consulta de documentos (ficha de caraterização da UC em estudo), considerando-se os objetivos definidos e a revisão da literatura sobre o tema (Cordner et al., 2012; Cox, 2012), bem como um estudo prévio (Antunes, 2016).

A instrução para a realização da narrativa foi formulada através de um apelo à apreciação das aulas, considerando as duas questões centrais do estudo: (1) a dinâmica em que as aulas decorreram; e (2) o conhecimento/aprendizagem conseguido sobre a metodologia qualitativa. De uma forma mais específica foi dada a seguinte instrução aos alunos:

Conte o que significou para si a experiência da disciplina de MI II, destacando:

- O que aprendeu.

- A importância da metodologia qualitativa.

- O mais útil.

- A dinâmica das aulas.

- Outros aspetos que julgue pertinentes e queira acrescentar.

A consulta de informação mais específica sobre a UC de MI II (de 7,5 ECTS), incidiu preferencialmente nos objetivos, programa e sistema de avaliação, os quais se descrevem a seguir, e de forma sucinta, conforme documento de trabalho da UC e disponível publicamente online na página da universidade:

(1) Objetivos: Estavam definidos da seguinte forma: 
"os alunos deverão ser capazes de enquadrar e caracterizar a metodologia de investigação qualitativa, descrever e aplicar técnicas básicas de recolha e análise de dados, comentar, planear e executar um projecto de investigação qualitativa";

(2) Programa: Apresentava-se organizado em quatro tópicos principais, iniciandose com uma parte introdutória à metodologia qualitativa e destacando-se a parte central, referente ao processo de investigação qualitativa, englobando conteúdos diversos (abordagens, os métodos de recolha, análise e interpretação de dados, bem como sobre a forma de apresentação e divulgação dos mesmos.

(3) Sistema de avaliação: Contemplava quatro elementos de avaliação: uma frequência individual e três trabalhos em grupo (uma resenha crítica de uma artigo qualitativo, um trabalho escrito (ensaio de estudo qualitativo) e a respetiva apresentação oral).

Além disso, era ainda facultada aos alunos a seguinte informação:

"A frequência incidirá sobre os conteúdos programáticos leccionados, requerendo uma compreensão, reflexão, integração e aplicação dos mesmos, a resenha crítica consistirá na realização de uma análise crítica de um estudo qualitativo, o trabalho escrito constará na elaboração de uma investigação e o teste oral remeterá para a apresentação do trabalho escrito."

As aulas da UC decorreram, por um lado, ao abordar conteúdo de índole mais teórico, com recurso ao método expositivo, conjugado com o método interrogativo, para a exposição e explicação das temáticas. Por outro lado, nas aulas de índole mais teórico-prática e de seminário, também se recorreu a outras metodologias de trabalho, destacando-se os métodos demonstrativo e ativo, promovendo o trabalho individual e em grupo. Como técnicas de trabalho utilizadas ao longo do semestre referem-se a exposição oral, as sessões de perguntas e respostas, a consulta de documentos (e.g., instrumentos de recolha de dados, artigos) acompanhada de análise e discussão, bem como o contacto com outros investigadores. Além disso, a promoção do trabalho autónomo do aluno privilegiou o ensaio e treino de competências de investigação, bem como o desenvolvimento do pensamento crítico, , principalmente através da realização e apresentação de trabalho(s) de investigação, o que, neste caso, se traduzia na realização dos trabalhos requeridos para avaliação .

\section{Procedimentos}

A lecionação da disciplina em estudo aconteceu no segundo semestre do ano letivo 2015/2016, a qual decorreu num total de 29 aulas (teórico-práticas e de seminário), distribuídas semanalmente em 2 aulas, uma com a duração de 120 minutos e a outra com duração de 90 minutos.

No decorrer do semestre, acompanhando o processo de aprendizagem, os alunos foram alvo de avaliação em três momentos: (1) através da realização, em grupo, de uma resenha crítica de um estudo qualitativo; (2) através da realização individual de 
uma prova escrita, apelando à reflexão e integração de conhecimentos; e (3) através da realização em grupo de um trabalho de investigação, resultando num texto escrito e respetiva apresentação oral.

$\mathrm{Na}$ última aula do semestre, mediante consentimento informado, foi solicitada aos alunos a elaboração de uma narrativa sobre a perceção que tinham acerca da importância da frequência da disciplina de MI II, sendo que aceitaram participar os doze alunos presentes na aula.

A instrução para a elaboração da narrativa foi dada aos alunos, sendo distribuída uma folha em branco para preenchimento individual, apelando à apreciação das aulas, considerando o significado atribuído à frequência desta UC, conforme instrução descrita atrás. Posteriormente, as narrativas foram alvo de análise de conteúdo (Bardin, 2013), considerando dois tópicos, já utilizados em estudo anterior (Antunes, 2016) como organizadores da narrativa, e definidos a priori, sendo estes tópicos decorrentes das duas questões centrais de investigação: (1) Dinâmica das Aulas (considerando aspetos sobre o funcionamento das aulas, sistema de avaliação e professor); e (2) Abordagem Qualitativa (considerando aspetos sobre a metodologia de investigação qualitativa).

Neste estudo, partiu-se igualmente, de forma dedutiva, das categorias identificadas no estudo já mencionado (Antunes, 2016), sendo que foram atribuídas as designações utilizadas sempre que se referiam a aspetos semelhantes e, portanto, se enquadravam na categoria. Nessa linha, para o tópico Dinâmica das Aulas consideraram-se as categorias: (1) Clima de aula e motivação; (2) Estratégias pedagógicas; (3) Papel do professor; e (4) Tempo de aulas. Para o tópico Abordagem Qualitativa tomaram-se as categorias: (1) Esclarecimento e conhecimento; (2) Rigor e validade; e (3) Experienciação. No entanto, apesar de se ter esse trabalho como referência, permitiu-se a exclusão de uma categoria (Tempo de aulas, no tópico Dinâmica das Aulas), pela sua não ocorrência, e a emergência de uma nova categoria (Ética, no tópico Abordagem Qualitativa).

Para manter o anonimato dos participantes, a sua identificação foi codificada através da atribuição da letra $A$ (de aluno) e de um número (atribuição de numeração a cada participante, por isso, de 1 a 12), sendo assim designados, ao longo deste texto, quando oportuna a transcrição de segmentos das narrativas.

\section{Resultados}

De forma a facilitar a leitura dos dados recolhidos apresentam-se os mesmos mediante os dois tópicos organizadores da narrativa e definidos a priori, ou seja, Dinâmica das Aulas e Abordagem Qualitativa, e as respetivas categorias (Figura 1), ilustrando-os com testemunhos dos participantes. 


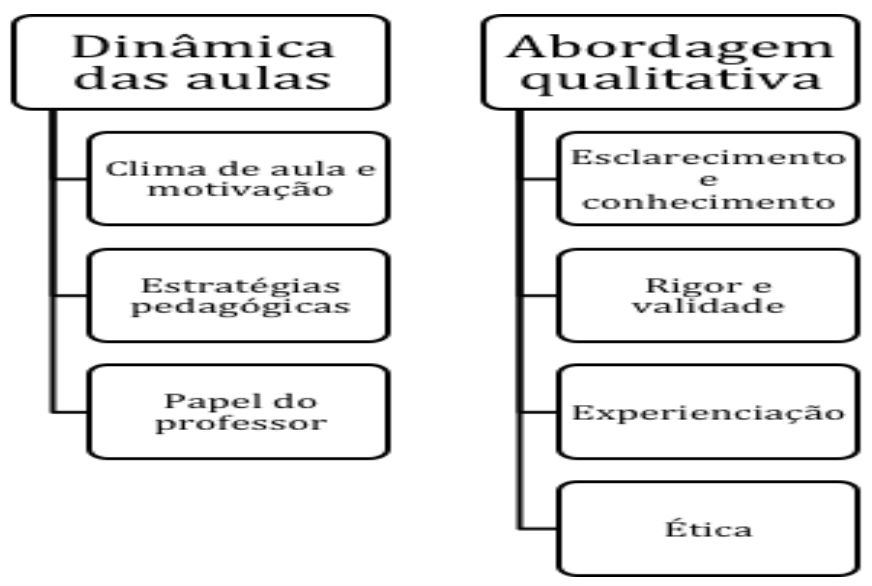

Figura 1: Tópicos e categorias emergentes nas narrativas

Quanto à Dinâmica das Aulas, sendo que este tópico remete para aspetos inerentes ao funcionamento das aulas e ao processo de ensino-aprendizagem, organizaramse 3 categorias:

(1) Clima de aula e motivação: Remetendo para a perceção do ambiente de sala de aula e de como este condicionava o investimento e dedicação dos alunos ("... despertou-nos para pesquisar mais sobre os assuntos falados..." - A1; "... as aulas tornaramse mais interessantes por terem sido mais interativas e práticas." - A2; "...gostei das aulas, achei a forma como era exposta a matéria bom e também muito bom o espaço que era sempre deixado à vontade para expor dúvidas e contribuir com opiniões muito produtivo, pois por vezes levou a que explorassemos mais, deixando também com curiosidade para explorar mais sobre o assunto em casa. " - A8;

(2) Estratégias pedagógicas: Nesta categoria, referente à metodologia de ensino utilizada nas aulas, os dados recolhidos permitiram verificar que as palavras dos alunos se referem, de uma forma geral, a 3 aspetos:

(a) adequação e satisfação com as técnicas e materiais utilizados ("...ajudou a assimilar a matéria, o facto de termos visualizado vídeos educativos, termos assistido a workshops relacionadas à matéria e, termos igualmente debatido os temas lecionados." - A2; "...se abordou e tentou por em prática alguns procedimentos próprios desta metodologia, por meio de trabalhos e exercícios feitos..." - A5; "A maneira como os conteúdos forma explorados, a disponibilização de materiais, os momentos que induziram a reflexão e a própria disponibilidade e métodos de ensino da docente são aspetos muito positivos e que suscitaram o interesse por este tipo de investigação e pela aquisição do conhecimento em geral. Também a realização de workshops..." A6; "Não foram aulas meramente expositivas." - A10); 
(b) a referência, com algum destaque, ao contacto direto com alguns investigadores e oportunidades de debate nas aulas ("... o facto de existirem pessoas convidadas... podermos participar e intervir, quer seja no decorrer das aulas ou na troca de opiniões sobre o filme, ou filmes, que foram passados nas aulas ou a partir dos trabaIhos de casa" - A9; "O debate é muito útil porque alguns juízos de valor que tínhamos em relação a este tipo de investigação eram desmistificados." - A11;); e

(c) a satisfação com o modelo de avaliação da UC ("...os trabalhos apresentados vieram complementar os conteúdos lecionados, por exemplo, foi relevante ouvir a experiência dos meus colegas ao realizar a entrevista e analisar a mesma. O trabalho foi muito exigente mas enriquecedor." - A1; "...considero que é benéfico existir uma frequência e um trabalho de grupo, dado que possibilita avaliar diferentes domínios." - A10; "E a realização de um trabalho "de campo" também permitiu termos noção de alguns procedimentos práticos e éticos necessários." - A11).

No entanto, surge também referência à necessidade de:

(a) algum treino mais intensivo e envolvimento na UC ("O que poderíamos ter feito mais nas aulas a nível de trabalhos práticos, talvez pequenos exercícios relativos à investigação qualitativa. - A7; "O tempo como sempre, é uma limitação, pois com outras cadeiras e outros envolvimentos nos divide sobre aquilo que alguns de nós poderiam aprofundar mais..." - A12); e

(b) outras temáticas nos trabalhos e a sua concretização ("...acho que poderia ser interessante se existissem mais temas para escolher nos trabalhos de grupo, ou então permitir que os alunos sugerissem alguns." - A9; "...provavelmente inverteria o tipo de trabalho de investigação..." - A12).

(3) Papel do professor: O testemunho dos alunos revela a perceção do papel crucial do professor não só como facilitador de acesso ao conhecimento, mas também na monitorização do investimento realizado ("...a forma como a professora organizou a informação sobre os trabalhos de grupo é uma ótima forma... tudo bem estruturado." - A3; "... a importância da literatura facultada pela docente, a qual ajudou muito na realização dos trabalhos que nos foram propostos e na aquisição de novos conhecimentos" - A6; "... acho muito bom e importante ver toda a abertura e disponibilidade da docente em ajudar os alunos." - A8).

No que se refere ao tópico Abordagem Qualitativa, sendo que este remete para a descoberta e aprofundamento que os alunos conseguiram acerca da metodologia qualitativa com a frequência das aulas, registaram-se 4 categorias:

(1) Esclarecimento e conhecimento: Remetendo, sobretudo, para 2 aspetos:

(a) a desmistificação de crenças ("...realmente todos nós vínhamos com uma ideia preconcebida acerca da dicotomia "quantitativo versus qualitativo"." - A2; "...ver a metodologia qualitativa com outros olhos..." - A8; "Foi-nos dada uma perspetiva diferente em relação ao que tínhamos aprendido até ao momento em relação à investigação, 
mais concretamente, à investigação qualitativa." - A12; "Consegui nesta unidade curricular ver a metodologia qualitativa com outros olhos..."); e

(b) a aquisição de novos conhecimentos sobre a investigação qualitativa ("...abriume as portas para a investigação qualitativa, método com o qual eu não estava familiarizada..." - A1; "...foi importante haver uma abordagem a este tipo de metodologia, não só porque permite estudar fenómenos de uma forma mais exaustiva e também idiossincrática, mas também porque será importante ter esses conhecimentos para a realização de estudos futuros" - A2; "...permitiram aprofundar um tipo de investigação com o qual não estava muito familiarizada, o que poderá ajudar em futuras investigações." - A6; "De facto, até este ano não tive essa experiência de forma tão aprofundada como agora." - A7).

No entanto, surge também a referência à necessidade de alguma continuidade e aprofundamento ("Devido à sua abrangência seria útil que houvesse uma continuidade tendo em conta as mais variadas formas de tratar os dados... seria mais útil haver... uma continuidade uma vez que fica o sentimento de falta de aprofundamento." - A5).

(2) Rigor e validade: Nas narrativas dos alunos emerge também, inerente ao processo de aprendizagem, a descoberta de que a investigação qualitativa também deve ser rigorosa e apresentar critérios de validade ("...no início senti dificuldade em compreender como assegurava a sua validade e rigor mas após as primeiras aulas comecei a desprender-me da investigação quantitativa..." - A1; "Aprendi que fazer um estudo utilizando a metodologia qualitativo é um processo exigente, mas é tão válido como fazer um estudo quantitativo..." - A9; "Aprendi também diferentes formas para o trabalho qualitativo ser rigoroso... foi compreender que podíamos ser rigorosos numa investigação qualitativa." - A11").

(3) Experienciação: Os alunos destacam ainda a valorização da oportunidade de experimentar na prática os conteúdos abordados (“...foi relevante ouvir a experiência dos meus colegas ao realizar a entrevista e analisar a mesma." - A1; "...poder colocar em prática o conhecimento adquirido em contexto de aula, permitindo um primeiro contacto (...) com as especificidades da metodologia qualitativa, assim como, do contacto com os entrevistados"- A3; "A realização dos trabalhos práticos foi muito enriquecedora, já que nos permitiu contactar, de forma mais próxima, com a metodologia qualitativa e obrigou-nos a refletir acerca de muitos aspetos a ela inerentes..." - A6).

(4) Ética: Emerge ainda a referência à postura ética do investigador ("As questões éticas foram também para mim de extrema importância" - A10; "...e a importância do código de ética e dos procedimentos éticos na investigação” - A11). 


\section{Discussão e Conclusões}

Os dados recolhidos permitem algumas considerações acerca dos tópicos em análise, ou seja, a Dinâmica das Aulas e a Abordagem Qualitativa. De uma forma geral, os alunos manifestam satisfação com a frequência desta unidade curricular e reconhecem a aquisição de conhecimento sobre a metodologia em estudo.

De uma forma mais específica, em relação ao tópico Dinâmica das Aulas, os dados parecem revelar que os alunos reconhecem que o clima de sala de aula permitiu a aprendizagem e contribuiu para a sua motivação, sendo que as estratégias pedagógicas utilizadas e o papel do professor foram apontados também como fatores importantes na aprendizagem. Neste ponto mantém-se a referência dos alunos ao contacto com outros investigadores, já registada em estudo anterior (Antunes, 2016), a qual aparece na literatura com um fator determinante na formação dos investigadores em iniciação (Cordner et al., 2012). Além disso, a pertinência atribuída à oportunidade de debate merece registo, uma vez que essa prática permite questionar crenças e criar situações de aprendizagem pela partilha e confronto de opiniões.

Simultaneamente, analisando o programa da UC e as categorias emergentes em relação à Dinâmica das Aulas, depreende-se a preocupação, ao longo da lecionação, de criar oportunidades aos alunos para apreenderem e adquirirem conhecimento e treino de competências de investigação qualitativa (Clarke \& Braun, 2013; Cordner, Klein, \& Baiocchi, 2012; Cox, 2012; Hansman, 2015; 2010, Terkildsen \& Petersen, 2015). Nessa linha de pensamento e como refere Amado (2014), importa referir também a disponibilidade e abertura por parte do professor a refletir sobre a sua prática pedagógica, permitindo questionar a eficácia do funcionamento da disciplina lecionada, o que este estudo também permite, contrapondo os dados com outros estudos realizados e contribuindo para a discussão sobre o ensino da metodologia qualitativa em Psicologia, também rara em Portugal (Hansman, 2015).

Não obstante a utilização de técnicas e materiais diversificados e a perceção positiva do desempenho do professor regista-se a referência à necessidade sentida por um aluno de mais atividades de treino, embora não especificando quais nem como, e uma outra referência à possibilidade de um maior envolvimento por parte dos alunos nas tarefas da disciplina. Neste trabalho, a categoria Tempo de aulas foi eliminada porque não se registou nenhuma ocorrência sobre esse factor a condicionar a aprendizagem como aconteceu noutro trabalho (Antunes, 2016).

Em relação ao tópico Abordagem Qualitativa, o testemunho dos alunos revela esclarecimento e desmistificação de crenças erradas acerca da metodologia qualitativa, 
como aliás se tem verificado noutros trabalhos (Clark \& Braun, 2013), bem como a aquisição de conhecimento que pode ser utilizado em trabalhos futuros (Wiggins, Gordon-Finlayson, Becker, \& Sullivan, 2016). Importa referir que a questão do rigor e da validade emerge nas palavras dos alunos, traduzindo a comparação estabelecida com a metodologia quantitativa, para eles mais conhecida, e o que entendiam como pesquisa científica (Clark \& Braun, 2013; Povee \& Roberts, 2014; Roberts \& Povee, 2014).

Importa mencionar a referência à consciência ética, crucial em investigação (Gomes, 2016), e a importância atribuída à oportunidade de poderem experienciar e treinar competências de investigação qualitativa de recolha e de análise dados. Aliás, consultando a informação sobre a disciplina verifica-se que no segundo trabalho os alunos, com supervisão do professor, realizaram e escreveram um texto (em formato de artigo) sobre a pesquisa realizada, como aliás acontece noutros casos descritos na literatura (Cordner et al., 2012). Este processo decorreu ao longo de algum tempo e procurou que os alunos fossem definindo uma proposta de investigação que depois concretizaram, sendo a orientação da docente importante pelo constante feedback, bem como a partilha e discussão em sala de aula, por exemplo, sobre as formas de acesso ao terreno, a coleta e análise de dados, permitindo a evolução dos alunos (Cox, 2012).

Um aspeto que pode testemunhar e contribuir para a perceção da aprendizagem conseguida pelos alunos nesta UC, e a sua motivação, não só neste ano letivo, mas em anos anteriores (Antunes, 2016), pode ser a referência ao facto de que, dois dos melhores trabalhos, foram ligeiramente reformulados e aceites para publicação em revista da área psicoeducativa (Fernandes, Manuel, \& Antunes, 2014; Lourenço, Câmara, Luís, \& Antunes, no prelo 2016).

No entanto, considerando a oportunidade de esclarecimento e conhecimento que a UC permitiu, regista-se uma referência à necessidade de continuidade e aprofundamento, devido à variedade e complexidade inerentes à metodologia qualitativa, quer em termos teóricos quer em termos práticos. Apesar de esta referência poder traduzir que alguns alunos poderão necessitar de mais tempo para se apropriarem de determinados conceitos ou para o treino de algumas competências também se pode referir que, na literatura, se encontra a referência a alguma dificuldade, por parte do professor, à gestão da lecionação dos temas e o tempo dedicado às questões epistemológicas e às diferentes abordagens (Clark \& Braun, 2013; Hansman, 2015; Terkildsen \& Petersen, 2015).

Apesar dos dados recolhidos serem satisfatórios algumas limitações podem ser apontadas. Em primeiro lugar, a instrução para a elaboração da narrativa decorreu da limitação apontada em estudo anterior (Antunes, 2016), onde se permitia uma narrativa mais aberta e, portanto, que diferentes alunos focassem diferentes aspetos conforme o que Ihes ocorria. Por isso, no presente trabalho foi-Ihes dada a indicação de 
abarcarem explicitamente determinados pontos na narrativa, o que, se por um lado, focou a atenção dos alunos nos mesmos pode, por outro lado, ter tido a desvantagem de não permitir maior flexibilidade nas narrativas. No entanto, parece-nos que tal não aconteceu porque se registou uma flutuação nas categorias encontrados (tanto a eliminação de uma categoria como o surgimento de nova categoria). Em segundo lugar, o presente estudo foi realizado na perspetiva do professor (e investigador) e resultado da sua preocupação pedagógica. Poderia, em trabalhos posteriores, contemplar-se, além da narrativa, a realização de entrevistas aos alunos e, por exemplo, a análise dos trabalhos finais e das suas apresentações orais.

Para finalizar, ainda que este trabalho se constitua como um estudo exploratório dado o estado da arte em Portugal sobre a temática, e centrado num caso específico, parece ter cumprido o objetivo que esteve na sua génese, ou seja, contribuir para a reflexão e discussão sobre o ensino da metodologia qualitativa em Psicologia, através da descrição de uma unidade curricular concreta e do testemunho dos alunos, evidenciando-se a pertinência quer dos aspetos pedagógicos envolvidos quer da metodologia qualitativa como forma de fazer ciência.

\section{Referências Bibliográficas}

Amado, J. C. (2014). A formação em investigação qualitativa: Notas para a construção de um programa. In A. P. Costa, F. N. Souza, \& D. N. Souza (Orgs.), Investigação qualitativa: Inovação, dilemas e desafios (vol. 1) (pp. 39-67). Oliveira de Azeméis: Ludomedia.

Antunes, A. P. (2016). Ensino de Investigação Qualitativa: Prática num Curso de Mestrado em Psicologia da Educação. In A. P. Costa, P. A. Castro, S. O. Sá, J. L. Carvalho, F. N. Souza, \& D. N. Souza (Eds.), Atas: Investigação Qualitativa na Educação (vol. 1, pp. 1075-1083). ISBN: ISBN: 978-9728914-58-5. [Disponível em http://proceedings.ciaiq.org/index.php/ciaiq2016/article/view/705/693, consultado em 25/10/2016].

Bardin, L. (2013). Análise de conteúdo. Lisboa: Edições 70.

Clarke, V., \& Braun, V. (2013). Teaching thematic analysis. Methods, 26(2), 120-123.

Cordner, A., Klein, P. T., \& Baiocchi, G. (2012). Co-designing and co-teaching graduate qualitative methods: An innovative ethnographic workshop model. Teaching Sociology, 40(3), 215-226.

Cox, R. D. (2012). Teaching qualitative research to practitioner-researchers. Theory Into Practice, 51, 129-136.

Culver, D. M., Gillbert, W., \& Sparkes, A. (2012). Qualitative research in Sport Psychology journals: The next decade 2000-2009 and beyond. The Sport Psychologist, 26, 261-281.

Denzin, N. K., \& Lincoln, Y. S. (2005). The SAGE handbook of qualitative research. Thousand Oaks: SAGE Publications.

Fernandes, E., Manuel, M., \& Antunes, A. (2014). A vida em cima da prancha: Um caso de excelência humana. Revista AMAzônica: Revista de Psicopedagogia, Psicologia Escolar e Educação, 13(1), 22-50. 
Gomes, C. A. (2016). Pressupostos éticos na investigação e produção científica em contexto académico: Dimensões científicas, metodológicas e institucionais. In J. R. Casanova, C. Bisinoto, L. S. Almeida (Eds.), Livro de Atas do IV Seminário Internacional Cognição, Aprendizagem e Desempenho. Braga: Centro de Investigação em Educação.

Goussinsky, R., Reshef, A., Yanay-Ventura, G., \& Yassour-Borochowitz, D. (2011). Teaching qualitative research for human services students: A three-phase model. The Qualitative Report, 16(1), 126-146. Retirado de http://www.nova.edu/ssss/QR/QR16-1/yassour-borochowitz.pdf

Hansman, C. A. (2015). Training librarians as qualitative researchers: Developing skills and knowledge. The Reference Librarian, 56, 4, 274-294. doi: 10.1080/02763877.2015.1057683

Humble, Á. M., \& Sharp, E. (2012). Shared journaling as a peer support in teaching qualitative research methods. The Qualitative Report, 17, 1-19. Retirado de http://www.nova.edu/ssss/QR/QR17/ humble.pdf

Lourenço, L., Câmara, E., Luís, L., \& Antunes, A. (2016). O aluno universitário e a pessoa com necessidades especiais: Percepções sobre a prática de voluntariado. Revista AMAzônica: Revista de Psicopedagogia, Psicologia Escolar e Educação, 18(2), 57-81. [Disponível em http://ieaa.ufam. edu.br/revistas/ojs/index.php/amazonica/article/view/63, consultado em 30/01/2017].

Page, R. N. (1997). A thought about curriculum in qualitative research methods. International Journal of Qualitative Studies in Education, 10(2), 171-174. doi: 10.1080/095183997237269

Poulin, K. L. (2007). Teaching qualitative research: Lessons from practice. The Counseling Psychologist, 35(3), 431-458. doi: 10.1177/0011000006294813

Povee, K., \& Roberts, L. D. (2014). Qualitative research in psychology: Attitudes of psychology students and academic staff. Australian Journal of Psychology, 66, 28-37. doi: 10.1111/ajpy.12031

Reynolds-Keefer, L. (2010). Improving the quality of experience journals: Training educational psychology students in basic qualitative methodology. Teaching Educational Psychology, 6(1), 20-32. Retirado de http://eric.ed.gov/?id=EJ894804

Roberts, L. D., \& Povee, K. (2014). A brief measure of attitudes towards qualitative research in psychology. Australian Journal of Psychology, 66, 249-256. doi: 10.1111/ajpy.12059

Rodríguez, R. M., Anguera, M. T., Suárez, E., León, O. G., \& Márquez, M. G. (2014, Setembro). El qué y el porqué de la metodología cualitativa en Psicología. Simpósio realizado no IX Congresso Iberoamericano de Psicologia e $2^{\circ}$ Congresso da Ordem dos Psicólogos Portugueses, Centro Cultural de Belém, Lisboa.

Terkildsen, T., \& Petersen, S. (2015). The future of qualitative research in psychology: A students' perspective. Integrative Psychological and Behavioral Science, 49, 202-206.

Wiggins, S., Gordon-Finlayson, A., Becker, S., \& Sullivan, C. (2016). Qualitative undergraduate project supervision in psychology: Current practices and support needs of supervisors across North East England and Scotland, Qualitative Research in Psychology, 13(1), 1-19, DOI: 10.1080/14780887.2015.1075641 
Ana Pereira Antunes

Faculdade de Artes e Humanidades, Universidade da Madeira

Funchal, Portugal

Centro de Investigação em Estudos da Criança, Universidade do Minho

Braga, Portugal

Email: aantunes@uma.pt

Correspondência

Ana Pereira Antunes

Faculdade de Artes e Humanidades, Universidade da Madeira

Campus Universitário da Penteada 9020-105 Funchal, Portugal

Data de submissão: Julho 2016

Data de avaliação: Outubro 2016

Data de publicação: Julho 2017 